\title{
Tunable topological charge vortex microlaser
}

\author{
Zhifeng Zhang ${ }^{1}$, Xingdu Qiao ${ }^{1}$, Bikashkali Midya ${ }^{2}, \mathrm{Kevin} \mathrm{Liu}^{2}$, Jingbo Sun ${ }^{3}$, Tianwei $\mathrm{Wu}^{2}$,
} Wenjing Liu ${ }^{2}$, Ritesh Agarwal ${ }^{2}$, Josep Miquel Jornet ${ }^{4}$, Stefano Longhi ${ }^{5,6}$, Natalia M. Litchinitser ${ }^{3}$, and Liang Feng ${ }^{2,1^{*}}$

${ }^{1}$ Department of Electrical and Systems Engineering, University of Pennsylvania, Philadelphia, Pennsylvania 19104, USA

${ }^{2}$ Department of Materials Science and Engineering, University of Pennsylvania, Philadelphia, Pennsylvania 19104, USA

${ }^{3}$ Department of Electrical and Computer Engineering, Duke University, Durham, North Carolina 27708, USA

${ }^{4}$ Department of Electrical and Computer Engineering, Northeastern University, Boston, Massachusetts 02115, USA

${ }^{5}$ Dipartimento di Fisica, Politecnico di Milano and Istituto di Fotonica e Nanotecnologie del Consiglio Nazionale delle Ricerche, Piazza L. da Vinci 32, Milano I-20133, Italy

${ }^{6}$ Instituto de Fisica Interdisciplinar y Sistemas Complejos IFISC (CSIC-UIB) - Palma de Mallorca, Spain

*Corresponding author: fenglia@,seas.upenn.edu

The orbital angular momentum (OAM) intrinsically carried by vortex light beams holds a promise for multi-dimensional high capacity data multiplexing, meeting the ever-increasing demands for information. Development of a dynamically tunable OAM light source is a critical step in the realization of the OAM multiplexing. By harnessing the properties of total momentum conservation, spin-orbit interaction, and optical non-Hermitian symmetry breaking, we demonstrate an OAM-tunable vortex microlaser, providing chiral light states of variable topological charges at a single telecommunication wavelength. The scheme of the non-Hermitiancontrolled chiral light emission at room temperature can be further scaled up for simultaneous multi-vortex emissions in a flexible manner. Our work provides a route for the development of the next generation of multi-dimensional OAM-spin-wavelength division multiplexing technology. 
In the digital era of proliferating connections between pervasive endpoints, the tremendously growing aggregated data traffic motivates the development of innovative optical communication technologies to sustain the required massive increase in information capacity. The current information infrastructure based on wavelength and time division multiplexing, together with other degrees of freedom of light including the amplitude, polarization, and phase, is, nevertheless, approaching a bottleneck. Fortunately, the full-vector nature of light provides another information dimension, namely, the angular momentum, to ease the upcoming information crunch. While the spin angular momentum (SAM) associated with the circular polarization of light is limited by two states $(\sigma=$ $\pm 1)$, the orbital angular momentum (OAM) of a vortex beam with an azimuthal phase dependence (i.e., $E \sim e^{i l \varphi}$, characterized by the topological integer $\left.l=0, \pm 1, \pm 2 \cdots\right)(1,2)$ creates a dimension of unlimited spatially distinguishable channels for data transmission. In addition to their potential for optical communications (3-6), optical vortex beams carrying OAM have also revolutionized several applications in the fields of optical manipulation $(7,8)$, imaging and microscopy $(9,10)$, and quantum information processing $(11,12)$.

Dynamically tunable vortex light sources have become essential to bring into reality these emerging photonic technologies based on the OAM degree of freedom. Traditional bulky optical components such as spiral phase plates and forked holograms $(2,13)$, or recently developed planar optical components including various metasurfaces (14-17) and silicon resonators (18), used for OAM beam generation, require an external input beam originating from a separate light source. Chip-scale microlasers offer a more compact and robust solution to obtain highly pure coherent vortex modes and have been recently investigated extensively (19-23). However, the demonstrated miniaturized vortex lasers at the telecommunication wavelengths so far lack reconfigurability, limited by their output of a pre-defined polarized OAM state per wavelength $(20,21)$. Nevertheless, the ongoing effort of OAM-SAM-wavelength (3-6) division multiplexing for multi-dimensional high capacity information processing requires the flexible generation and versatile manipulation of different OAM and SAM states at the same wavelength to fully take advantage of the orthogonality of OAM modes, which is not yet accessible by state-of-the-art microscale devices.

Here, we use the spin-orbit coupling, based on the conservation of the sum of the OAM and transverse spin, to precisely maneuver the chiral light states in microring lasers. The ability to simultaneously and cohesively manipulate both the SAM and OAM degrees of freedom can couple the local spin with orbital oscillation of optical cavity modes, thereby leveraging richer functionalities in vortex light generation $(23,24)$. Spin-orbit coupling can enable effective control of the OAM handedness arising from the chiral symmetry of $\pm|l|$ wavefunctions, featuring the 
ability to flexibly generate mutli-vortex emissions of variable $|l|$ OAMs in the full angular momentum space. Although it is straightforward to switch the chirality depending on the direction of input light in a passive microring resonator (18), a sophisticated active, robust strategy is required to selectively break the chiral symmetry intrinsically associated with a microlaser, yielding flexible control of spin-orbit interaction. Optical control of spin-orbit interaction and its induced chiral light emission have been demonstrated through spin polarized gain generation in a semiconductor polaritonic system (23), however in such a device a cryogenic environment is required, which is not suitable for practical integration in current information systems. To create a robust yet reconfigurable spin-orbit coupling in an ambient temperature environment, we demonstrate a microlaser system in which spin-orbit interaction is controlled by an externally applied non-Hermitian coupling between the cavity modes [Fig 1]. Strategic nonHermitian symmetry breaking facilitates the lifting of degeneracy between two spin-orbit coupled states, of which the spin is locked to the orbit direction, in a controllable manner and favors the lasing of an OAM state of desirable chirality. The spin-orbit interaction, together with the conservation of the total angular momentum, is further exploited to alter the emitted OAM state by converting the SAM into OAM, introducing the additional tunability with variable topological charges at the same wavelength. This scheme can be further scaled up to simultaneously generate multiple laser vortices with an imaginary gauge (26) conducted in the coupled system.

We consider a microring resonator supporting the two degenerate clockwise (U) and counter clockwise $(\circlearrowleft)$ whispering gallery modes (WGMs) on a III-V semiconductor platform with a $200 \mathrm{~nm}$ thick InGaAsP multiple quantum well layer, coupled with an additional bus waveguide of InGaAsP with two control arms which enables the indirect coupling between the two modes [Fig. 1 and Fig. 2]. By finely tuning the aspect ratio of the cross section geometry of the waveguide, the transverse spin in the evanescent tail of guided light is engineered to be 1, achieved with equal amplitudes of the radial and azimuthal components of the electric field (27). Note that the absolute value of transverse spin generated in this scheme cannot exceed 1 . The spin-orbit interaction consequently couples the right/left-hand circular polarization $(\downarrow / \uparrow)$ with the $\circlearrowright / \cup$ mode, leading to spin-orbit locking for the light circulating in the microring. The degeneracy between these two counter-circulating states is broken by introducing non-Hermitian mode coupling via the bus waveguide. By optically pumping one of the waveguide arms to create gain (generated through pumping) and loss (intrinsic material loss without pumping) contrast, an effective asymmetric coupling between the counter-propagating WGMs in the microring is obtained. The Hamiltonian of the non-Hermitian controlled microring resonator can effectively be described as 


$$
H_{\mathrm{eff}}=\left(\begin{array}{cc}
\omega_{\circlearrowright} & \kappa e^{-\gamma} e^{+\gamma_{L}} \\
\kappa e^{-\gamma} e^{+\gamma_{R}} & \omega_{\circlearrowleft}
\end{array}\right)
$$

where $\omega_{\circlearrowright / \circlearrowleft}$ is the eigen frequency of the two degenerate modes, $\kappa$ denotes the coupling between the cavity modes without any gain/loss in the bus waveguide, $-\gamma$ describes the single pass attenuation due to the intrinsic material loss, and $+\gamma_{R}\left(+\gamma_{L}\right)$ represents the single pass amplification from the optical pumping applied to the right (left) control arm, respectively. This effective Hamiltonian can also be transformed into a parity-time symmetric form by using the basis of $\omega_{\circlearrowright}+i \omega_{\circlearrowleft}$ and $\omega_{\circlearrowright}-i \omega_{\circlearrowleft}$ states (27). In the absence of optical pumping on the control $\operatorname{arm}\left(\gamma_{L}=\gamma_{R}=0\right)$, the overall coupling strength between $\circlearrowright$ and $U$ modes approaches zero because of the strong intrinsic material loss of the InGaAsP multiple quantum well $(\gamma \gg 1)$ [Fig. 3A]. When the left (or right) control arm is selectively pumped, the intrinsic material loss on the left (or right) is then overcome by net optical gain, i.e., $\gamma_{L}>\gamma$ (or $\left.\gamma_{R}>\gamma\right)$. On the other hand, the unpumped right (left) side of the waveguide remains lossy. Due to spin-orbit locking selective pumping results in a unidirectional coupling between the $\circlearrowright$ and $U$ modes, breaking the chiral symmetry and facilitating an effective means to controlling the chirality of the mode [Fig. 3C and Fig. 3E]. Under this condition, the microlaser effectively operates at an exceptional point (20). Unidirectional emission is robust against instabilities induced by nonlinearities above the laser threshold (28), and is essential to realize our single-frequency microlaser with reconfigurable OAM via spin-orbit coupling.

The non-Hermitian controlled microlaser was designed such that the angular momentum carried by the WGM inside the resonator is extracted into free space by introducing periodic angular scatters inside the inner sidewall [Fig. 2]. The angular scatters are located at $\theta_{q}=2 \pi q / M$ and carry a local phase of $\varphi_{\text {local }, q}=2 \pi C q(N-M) / M$, where $q \in\{0, M-1\}, C=\operatorname{sgn}(\sigma)$ for the $U$ and $\circlearrowright$ modes, respectively, and $N$ is the order of the WGM. By accounting for the rotation of local coordinates with respect to the global coordinate, the extracted global phase can be expressed as $\varphi_{\mathrm{global}, q}=2 \pi C[q(N-M)-q] / M$. Consequently, the linearly varying phase distribution creates an OAM emission with a topological charge of $l=C(N-M-1)$ and a total angular momentum of $J=C(N-M)$. The corresponding vortex emission, containing both SAM and OAM as well as their associated chirality, can be represented as ||$l|, \operatorname{sgn}(l), \sigma\rangle$. In our experiment, the microlaser cavity has a diameter of $7 \mu \mathrm{m}$ and a width of $0.65 \mu \mathrm{m}$, which supports a WGM of order $N=34$ and scatter number $M=32$ for a total angular momentum of $|J|=2$.

To better configure the non-Hermitian-controlled chirality and its resulting spin-orbit interaction, two synchronized pump beams from a nanosecond laser were projected: one onto the microlaser cavity to carry out the lasing, and the other to selectively pump one of the control arms 
to manipulate the chirality of the lasing (27). The chiral symmetry of the system is observed in the absence of control pump [Fig. 3A]. The emission from the resonator showed right/left-handed circularly polarized components of nearly balanced intensities without a net spin angular momentum [Fig. 3B]. This reveals the absence of net non-zero angular momentum resulting from the coexistence of the two degenerate $U / \cup$ modes. To probe the vortex nature of laser radiations, the emitted beam was split into two identical beams and were subsequently interfered with each other with a slight offset in the horizontal direction. The acquired interferogram shows no significant fringe mismatch or discontinuity, indicating no significant phase changes near the center of the beam, which confirms a net zero OAM charge [Fig. 3B]. In stark contrast, when the control beam was switched on while illuminating only the right control arm (Fig. 3C), unidirectional laser oscillation arose in the $U$ mode. The emission of nearly perfect left-handed circular polarization was consequently observed, revealing the unidirectional power circulation of the $\cup$ mode inside the microring and spin-orbit locking. Due to the phase singularity, the phase distribution changes drastically near the center of the emitted laser beam while maintaining relatively uniform distribution at the outer regions. The corresponding self-interference pattern, therefore, shows a pair of inverted forks, verifying the topological charge of OAM laser emissions. In each fork, a single fringe split into two, corresponding to an OAM charge of +1 [Fig. 3D]. On the contrary, when the control beam was only applied onto the left control arm (Fig. 3E), a nearly perfect right-handed circularly polarized emission was observed instead, demonstrating the non-Hermitian-controlled switching of chirality via spin-orbit locking. The orientations of the forks were reversed compared with the previous condition, where the single fringe still split into two but in the opposite direction, verifying an OAM charge of -1 [Fig. 3F].

Additional tunability of the OAM charge through the conservation of the total angular momentum in the spin-to-orbit conversion was demonstrated with the vortex microlaser using a radial polarizer that preserves the rotational symmetry of the emitted laser beam (27). The radial polarizer allows the transmission of only radially polarized light and thus converts circularly polarized light into a linearly polarized beam in a polar coordinate. Since the radial polarizer does not break the rotational symmetry, the total angular momentum, i.e., $J=l+\sigma$, of light must remain conserved. For a given $J, l$ can therefore be controlled via the manipulation of $\sigma$. The new OAM state with $l= \pm 2$ were achieved here after the transfer of $\sigma= \pm 1$ SAMs into the demonstrated $l= \pm 1$ OAMs, respectively, when the laser beam was passed through the polarizer. The selfinterference interferograms were captured to analyze the vortex reconfiguration of the emitted beams. A pair of inverted forks with 3 fringes were observed, revealing a phase winding of $4 \pi$ at the center, which confirmed the OAM charge $l= \pm 2$ (top and bottom panels of Fig. 4A and Fig. 
4B). Altogether, the non-Hermitian controlled vortex microlaser is capable of producing 5 different OAM states ranging in $l=-2,-1,0,+1,+2$ in a reconfigurable manner. Notably, the lasing of all these OAM states occurred at a 'single' wavelength at $1492.6 \mathrm{~nm}$ (Fig. 4C), potentially providing 5 orthogonal channels for encoding information. With the intrinsic orthogonality of the OAM modes, the achievable data rate of a communication system can be increased by up to 5 times (27). The switching time of spin-orbit coupling is in principle limited by the semiconductor optical response with potential to reach ultrafast OAM switching in the picosecond scale (29), so that our reconfigurable OAM microlaser, besides of emitting at fixed wavelength, is much faster than those based on thermo-optic control of WGM resonances ( $\mu$ s to ms timescale). More importantly, our non-Hermitian spin-orbit mode control scheme is scalable by cascading sequential microrings to simultaneously generate multiple laser vortices; experimental results are given in (27).

Our tunable OAM microlaser is capable of emitting vortex beams of 5 different topological charges at room temperature. The non-Hermitian manipulation of chiral spin-orbit interaction offers fundamentally new functionality of controllable vortex light emission in a scalable way. The non-Hermitian control of spin-orbit interactions and thus OAM emissions is general and compatible with conventional electrical pumping schemes where standard p-i-n semiconductor configurations are exploited for current injection to excite optical gain (30). The toolbox of generating various vortex light at a single wavelength holds the promise for future development of multi-dimensional OAM-SAM-wavelength division multiplexing for highdensity data transmission in classical and quantum regimes. Additionally, dynamic switching between different OAM modes in time can further increase the security of wired and wireless communication networks (27). 


\section{Reference:}

1. L. Allen, M. W. Beijersbergen, R. J. C. Spreeuw, J. P. Woerdman, Orbital angular momentum of light and the transformation of Laguerre-Gaussian laser modes. Phys. Rev. A 45, 8185 (1992).

2. A. M. Yao, M. J. Padgett. Orbital angular momentum: origins, behavior and applications, $A d v$. Opt. Photonics 3, 161-204 (2011).

3. J. Wang, et al., Terabit free-space data transmission employing orbital angular momentum multiplexing. Nat. photonics 6, 488 (2012).

4. N. Bozinovic, et al., Terabit-scale orbital angular momentum mode division multiplexing in fibers. Science 340, 1545-1548 (2013).

5. I. M. Fazal, et al., 2 Tbit/s free-space data transmission on two orthogonal orbital-angularmomentum beams each carrying 25 WDM channels. Opt. Lett. 37, 4753-4755 (2012).

6. A. E. Willner, et al. Optical communications using orbital angular momentum beams. $A d v$. Opt. Photonics 7, 66-106 (2015).

7. H. He, M. E. J. Friese, N. R. Heckenberg, H. Rubinsztein-Dunlop, Direct observation of transfer of angular momentum to absorptive particles from a laser beam with a phase singularity. Phys. Rev. Lett. 75, 826 (1995).

8. M. Padgett, R. Bowman, Tweezers with a twist. Nat. photonics 5, 343 (2011).

9. L. Torner, J. P. Torres, S. Carrasco, Digital spiral imaging. Opt. Express 13, 873-881 (2005).

10. C. Maurer, A. Jesacher, S. Bernet, M. Ritsch - Marte, What spatial light modulators can do for optical microscopy. Laser Photonics Rev. 5, 81-101 (2011).

11. A. Mair, A. Vaziri, G. Weihs, A. Zeilinger, Entanglement of the orbital angular momentum states of photons. Nature 412, 313 (2001).

12. J. Leach, et al., Quantum correlations in optical angle-orbital angular momentum variables. Science 329, 662-665 (2010).

13. D. Naidoo, et al., Controlled generation of higher-order Poincaré sphere beams from a laser. Nat. Photonics 10, 327 (2016).

14. N. Yu, et al., Light propagation with phase discontinuities: generalized laws of reflection and refraction. Science 334, 333-337 (2011).

15. D. Lin, P. Fan, E. Hasman, M. L. Brongersma, Dielectric gradient metasurface optical elements. Science 345, 298-302 (2014).

16. M. I. Shalaev, et al., High-efficiency all-dielectric metasurfaces for ultracompact beam manipulation in transmission mode. Nano Lett. 15, 6261-6266 (2015).

17. C. W. Qiu, Y. Yang, Vortex generation reaches a new plateau. Science 357, 645-645 (2017). 
18. X. Cai, et al., Integrated compact optical vortex beam emitters. Science 338, 363-366 (2012).

19. B. Bahari, et al., Topological lasers generating and multiplexing topological light. arXiv: 1904.11873 (2019).

20. P. Miao, et al., Orbital angular momentum microlaser. Science 353, 464-467 (2016).

21. W. E. Hayenga, et al., Direct generation of tunable orbital angular momentum beams in microring lasers with broadband exceptional points. ACS Photon. 6, 1895-1901 (2019).

22. N. C. Zambon, et al., Optically controlling the emission chirality of microlasers. Nat. Photonics 13, 283 (2019).

23. K. Y. Bliokh, F. J. Rodríguez-Fortuño, F. Nori, A. V. Zayats, Spin-orbit interactions of light. Nat. Photonics 9, 796 (2015).

24. Z. Shao, J. Zhu, Y. Chen, Y. Zhang, S. Yu, Spin-orbit interaction of light induced by transverse spin angular momentum engineering. Nat. Commun. 9, 926 (2018).

25. S. Longhi, L. Feng, Mitigation of dynamical instabilities in laser arrays via non-Hermitian coupling, APL Photon. 3, 060802 (2018).

26. S. Longhi, Non - Hermitian Gauged Topological Laser Arrays. Ann. Phys. (Berl.). 530, 1800023 (2018).

27. Materials, methods, and additional information are available as supplementary materials.

28. S. Longhi, L. Feng, Unidirectional lasing in semiconductor microring lasers at an exceptional point, Photon. Res. 5, B1 (2017).

29. C. Huang, et al., Ultrafast control of vortex microlasers. Science 367, 1018-1021 (2020).

30. J. Zhang, et al., An InP-based vortex beam emitter with monolithically integrated laser. Nat. Commun. 9, 1-6 (2018).

31. O. Edfors, A. J. Johansson, Is orbital angular momentum (OAM) based radio communication an unexploited area? IEEE Trans. Antennas Propag. 60, 1126-1131 (2011).

32. M. Andersson, E. Berglind, G. Björk, Orbital angular momentum modes do not increase the channel capacity in communication links. New J. Phys. 17, 043040 (2015).

33. Z. Zhang, et al., The capacity gain of orbital angular momentum based multiple-inputmultiple-output system. Sci. Rep. 6, 25418 (2016).

34. J. Ma, et al., Security and eavesdropping in terahertz wireless links. Nature 563, 89 (2018).

35. L. Liang, W. Cheng, W. Zhang, H. Zhang, Mode hopping for anti-jamming in radio vortex wireless communications. IEEE Trans. Veh. Technol. 67, 7018-7032 (2018).

\section{ACKNOWLEDGEMENTS}


Funding: We acknowledge the support from U.S. Army Research Office (ARO) (W911NF-19-10249) and the National Science Foundation (NSF) (ECCS-1932803, ECCS-1846766, ECCS1842612, CMMI-1635026, DMR-1809518, and IIP-1718177). This research was partially supported by NSF through the University of Pennsylvania Materials Research Science and Engineering Center (MRSEC) (DMR-1720530). This work was carried out in part at the Singh Center for Nanotechnology, which is supported by the NSF National Nanotechnology Coordinated Infrastructure Program under grant NNCI-1542153. Author contributions: Z.Z. and L.F. conceived the project and conducted the design. Z.Z., B.M., T.W., K.L., J.S., N.M.L. and S.L. constructed the theoretical model and numerical simulations. X.Q., T.W., and W.L. fabricated the samples. Z.Z. and K.L. performed the measurements. Z.Z., B.M., L.F., J.S., N.M.L., S.L., R.A. and J.M.J. carried out data analyses. J.M.J. created the model for communication based on dynamically tunable OAMs. All authors contributed to manuscript preparation and discussion. Competing interests: The authors declare no competing interests. Data and materials availability: All data are available in the manuscript or the supplementary materials. 


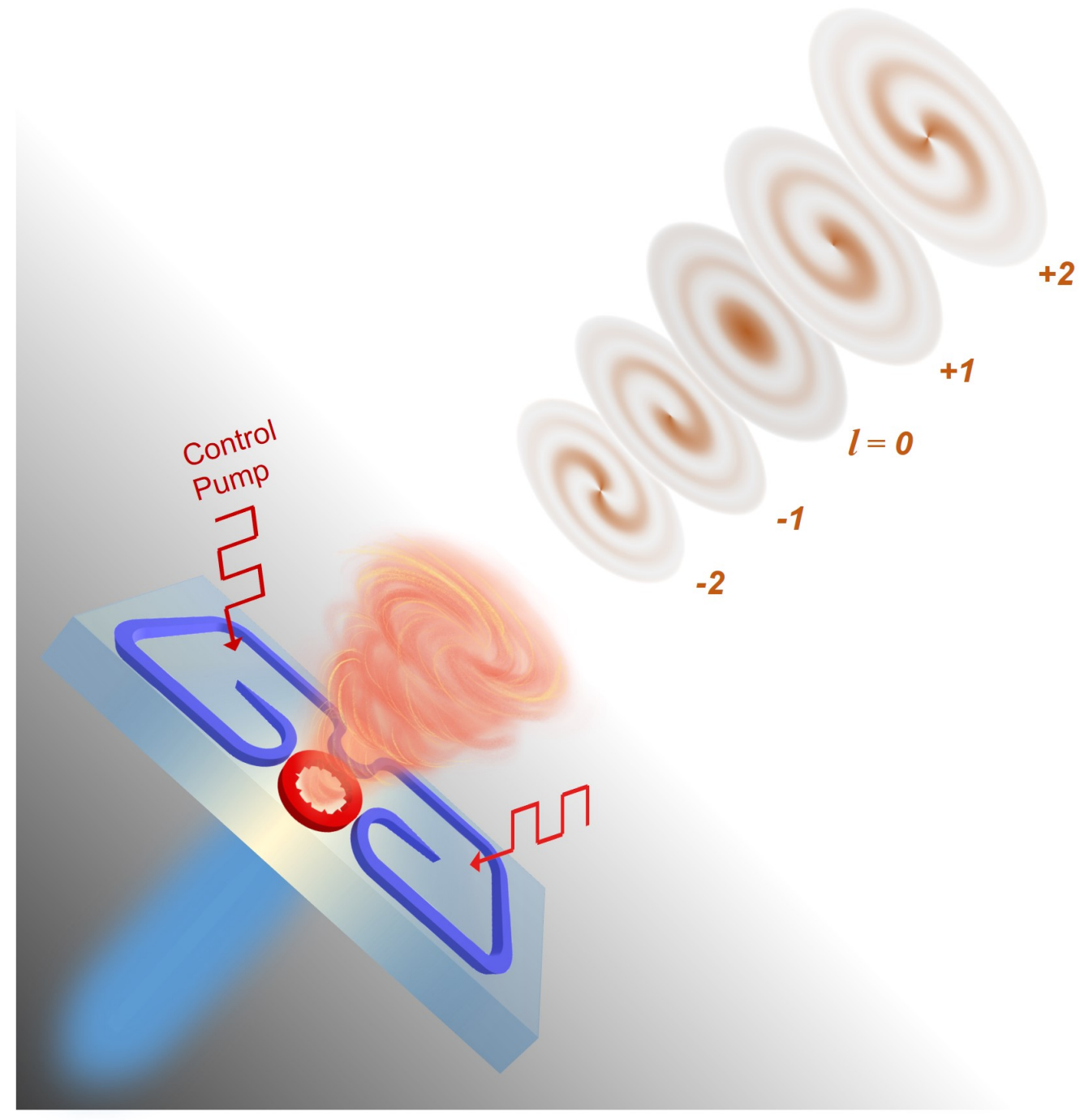

Fig. 1: Schematic of non-Hermitian controlled vortex microlaser. The non-Hermitian interaction mediated by the externally applied control pump on the bus waveguide can flexibly be switched for the emission of OAM states with desirable chirality from the spin-orbit engineered microring. 


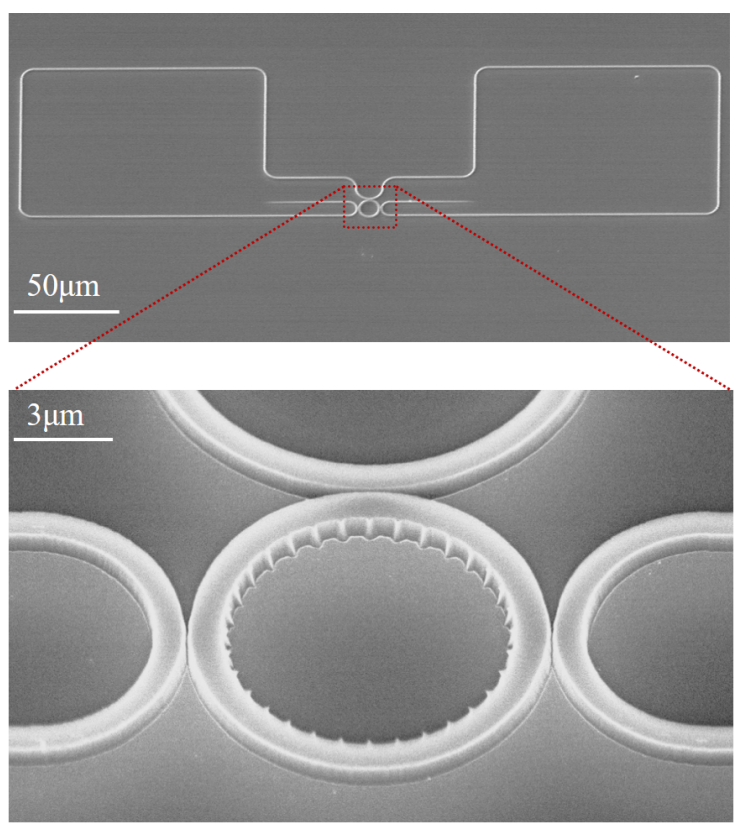

Fig. 2: Scanning electron microscope images of the tunable vortex microlaser. On an InGaAsP multiple quantum well platform, the microlaser consists of a main microring cavity coupled to an external feedback loop that enables the on-demand chiral control by selective pumping and thus enforces the unidirectional coupling between the two circulating modes in the microring. The angular grating is patterned on the inner side wall of the microring to produce the vortex laser emission of variable topological charges. 
A

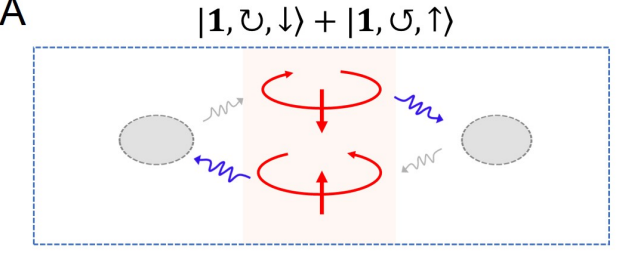

C

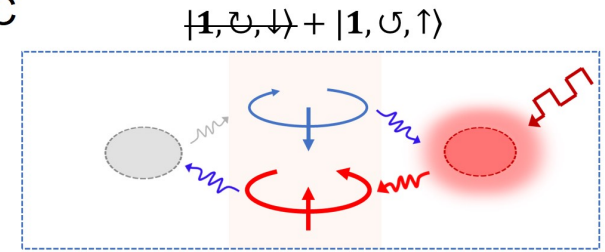

$\mathrm{E}$



B



$\mathrm{D}$

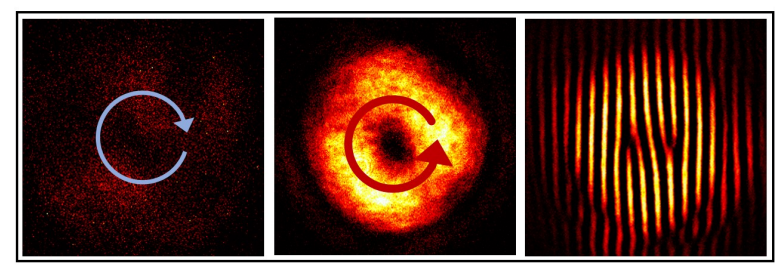

F



Fig 3: Non-Hermitian chiral interactions and experimental characterization of the lasing emission. (A) In the absence of non-Hermitian interaction, both the cavity modes represented by ||$l|, \operatorname{sgn}(l), \sigma\rangle$, with $\operatorname{sgn}(l)= \pm 1$ denoting the $\mathrm{CCW}(\circlearrowleft)$ and $\mathrm{CW}(\circlearrowright)$ oscillations, respectively, and $\sigma= \pm 1$ denoting the left $(\uparrow)$ and right $(\downarrow)$ circularly polarized light, are favored for lasing action. (B) Corresponding experimentally measured output emissions and off-center self-interference pattern of both the states. (C, D) Lasing emission of left-chiral mode measured after the introduction of non-Hermitian interaction applied by the right-side control pump. (E, F) Lasing emission of right-chiral mode measured after the reversing the non-Hermitian interaction applied by the left-side control pump. The shaded rectangular area in the left-hand panels denotes the microring cavity, and the shaded elliptical areas refers as non-Hermitian control arms. The red (gray) arrow outside the cavity denotes the gain (loss) feedback, while the blue arrow represents energy outcoupled from the cavity into the environment. Because of the unidirectional gain feedback, the cavity mode in red color is selectively favored for chiral lasing action, while the other cavity mode in blue is suppressed. 

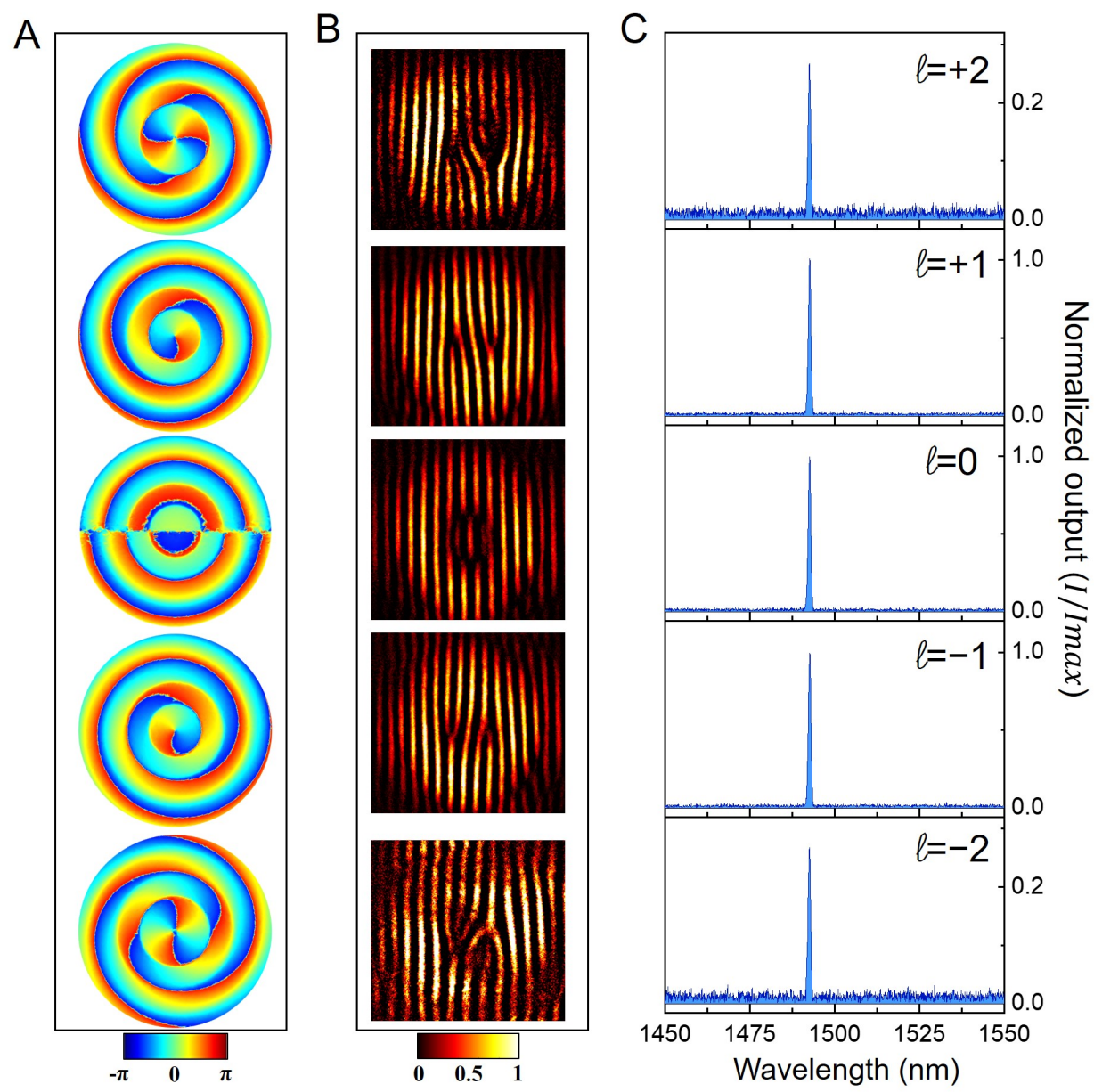

Fig 4: Experimental characterization of different OAM states at a fixed wavelength. (A) Simulated phase distributions of OAMs of charges $l=0, \pm 1$, and \pm 2 , showing the helical phase winds $2 l \pi$ around the center. The OAMs of lower three orders were achieved by spin-orbit locking and higher order OAMs were obtained by the spin-to-orbit coupling: $(\sigma, l)=( \pm 1, \pm 1)$ to $(\sigma, l)=(0, \pm 2)$. In both cases the chiral symmetry was broken by controllable non-Hermitian mode coupling. (B) Corresponding experimental results showing the off-center self-interference patterns (Figures are enhanced for better visualization). While no significant fringe mismatch was observed for $l=0$ state, a pair of inverted forks were observed for non-zero OAMs. A single fringe splits into two/three indicating a phase wind of $2 \pi, 4 \pi$ around the center singularity point confirming the OAM of $|l|=1,2$ respectively. The forks associated with opposite chirality were in opposite directions. (C) Measured lasing spectra show all five OAM lasing peaks located at 
$1492.6 \mathrm{~nm}$, implying that the vortex microlaser can generate single-frequency vortex beams of variable OAMs simultaneously. 